\title{
Variações na demanda de energia metabólica de juvenis de Haemulon steindachneri (Perciformes, Haemulidae) em função da temperatura
}

\author{
(Variations of metabolic energy demand of juveniles of \\ Haemulon steindachneri(Perciformes, Haemulidae) as function of temperature)
}

\author{
Arthur José da Silva Rocha; Vicente Gomes; Phan Van Ngan \& \\ Maria José de Arruda Campos Rocha Passos \\ Instituto Oceanográfico da Universidade de São Paulo \\ (Caixa Postal 66149, 05315-970 São Paulo, SP, Brasil)
}

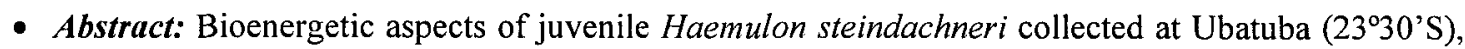
SP, Brazil, were evaluated as function of body weight and temperature $\left(15^{\circ} \mathrm{C}, 20^{\circ} \mathrm{C}\right.$ e $\left.26^{\circ} \mathrm{C}\right)$ through the oxygen consumption and ammonia excretion analyses. $\mathrm{Q}_{10}$ values and elevations a of the regression of oxygen consumption on wet weight at $15^{\circ} \mathrm{C}$ and $20^{\circ} \mathrm{C}$ were not significantly different. On the other hand, $Q_{10}$ and elevations a were significantly different between $20^{\circ} \mathrm{C}$ and $26^{\circ} \mathrm{C}$. It is suggested that $15-20^{\circ} \mathrm{C}$ is a temperature range of thermal metabolic independence for $H$. steindachneri, and this might be related to physiological adaptation to the environmental constraints. Daily energetic costs of routine metabolism were calculated from oxygen consumption data and other parameters of the bioenergetic equation were estimated following a model proposed in the literature. For a fish of $20 \mathrm{~g}$ and $70 \mathrm{~g}$ body weight (the minimum and maximum common weights at the 3 tested temperatures), it was estimated that their daily energetic demand at $15^{\circ} \mathrm{C}$ were 868.83 $\mathrm{cal} /$ day and $3168.65 \mathrm{cal} / \mathrm{day}$; at $20^{\circ} \mathrm{C}$ are $893.22 \mathrm{cal} /$ day and $2654.04 \mathrm{cal} / \mathrm{day}$ and at $26^{\circ} \mathrm{C}$ were $1390.30 \mathrm{cal} /$ day and $5046.61 \mathrm{cal} /$ day. Results contribute to the understanding of the ecological role of the species and its adaptation to the environment.

- Resumo: Avaliaram-se aspectos da bioenergética de $H$. steindachneri, em função do peso e da temperatura $\left(15^{\circ} \mathrm{C}, 20^{\circ} \mathrm{C}\right.$ e $\left.26^{\circ} \mathrm{C}\right)$, através do consumo de oxigênio e da excreção de amônia de exemplares jovens coletados na região costeira de Ubatuba ( $\left.23^{\circ} 30^{\prime} \mathrm{S}\right), \mathrm{SP}$, Brasil. Os valores de $\mathrm{Q}_{10} \mathrm{e}$ das elevações a das regressões entre o consumo de oxigênio e o peso úmido não revelaram diferenças significativas entre os dados obtidos nas temperaturas de $15^{\circ} \mathrm{C}$ e $20^{\circ} \mathrm{C}$. Por outro lado, os valores de $\mathrm{Q}_{10}$ e das elevações a entre $20^{\circ} \mathrm{C}$ e $26^{\circ} \mathrm{C}$ foram significativamente diferentes. A excreção de amônia aumentou significativamente entre as três temperaturas testadas, conforme demonstram as análises do $\mathrm{Q}_{10}$ e das elevações a. Sugere-se que o intervalo de temperatura de $15^{\circ} \mathrm{C}$ a $20^{\circ} \mathrm{C}$ seja uma faixa de independência térmica do metabolismo de $H$. steindachneri, o que, provavelmente, está relacionado a adaptação fisiológica às condições ambientais. A partir dos dados de consumo de oxigênio, calcularam-se os gastos energéticos diários com o metabolismo de rotina e estimaram-se, também, os demais parâmetros da equação bioenergética segundo modelo proposto na literatura. Para peixes de $20 \mathrm{~g}$ e $70 \mathrm{~g}$ de peso (mínimo e máximo comuns às 3 temperaturas testadas) estimouse que as necessidades energéticas diárias a $15^{\circ} \mathrm{C}$ foram $868,83 \mathrm{cal} / \mathrm{dia}$ e $3168,65 \mathrm{cal} / \mathrm{dia}$; a $20^{\circ} \mathrm{C}$ $893,22 \mathrm{cal} / \mathrm{dia}$ e $2654,04 \mathrm{cal} / \mathrm{dia}$ e a $26^{\circ} \mathrm{C}$ foram $1390,30 \mathrm{cal} / \mathrm{dia}$ e $5046,61 \mathrm{cal} / \mathrm{dia}$. Os resultados obtidos contribuem para o conhecimento do papel ecológico da espécie e de seus mecanismos de adaptação aos fatores ambientais.

- Descriptors: Haemulon steindachneri, Bioenergetic, Metabolism, Oxygen Consumption, Ammonia excretion.

- Descritores: Haemulon steindachneri, Bioenergética, Metabolismo, Consumo de Oxigênio, Excreção de amônia. 


\section{Introdução}

A plataforma continental ao largo de Ubatuba ( $23^{\circ} 30^{\prime}$ S), SP, Brasil, tem sido estudada pelo Instituto Oceanográfico da Universidade de São Paulo sobre diversos aspectos. Sua complexa estrutura biótica é influenciada por variações físico-químicas, tais como de temperatura e disponibilidade de nutrientes, decorrentes, principalmente, de um marcante padrão sazonal de circulação de massas d'água que afeta a produtividade, a distribuição e a dinâmica trófica das comunidades marinhas costeiras (Aidar et al., 1993).

Os indivíduos da espécie Haemulon steindachneri, conhecidos vulgarmente como "corcoroca-de-boca-larga", são representantes permanentes da ictiofauna da plataforma interna da região. Os dados existentes sobre sua biologia e distribuição indicam que estes animais estão entre os mais abundantes nas áreas rasas e de costões rochosos, possuindo um papel ecológico de destaque, essencial para manter a integridade do ecossistema (Cunningham, 1983; Furia, 1996; Rocha, 1997). Saul (1999) verificou ser essa espécie uma das dominantes de recifes artificiais implantados na Enseada das Palmas, Ubatuba. Distribui-se ao longo dos oceanos Pacífico e Atlântico tropicais, alimentando-se de pequenos invertebrados (Menezes \& Figueiredo, 1980). Embora não se conheçam em detalhes os hábitos dos indivíduos dessa espécie, os Haemulidae apresentam caracteristicamente um hábito migratório diário associado aos períodos de alimentação (Ogden \& Ehrlich, 1977; Estrada, 1986; Burke, 1995). A migração parece estar sincronizada às variações de luminosidade que ocorrem ao amanhecer e ao anoitecer (McFarland et al.,1979; Quinn \& Ogden, 1984), tendo como possíveis vantagens a repartição eficiente da área de "pastagem" e a redução da vulnerabilidade à predação (Lokkeborg \& Fernös, 1999). Esse comportamento contribui para a distribuição de energia e de nutrientes nos recifes e seus entornos (Meyer \& Schultz, 1985; Bray et al., 1986; Clarke, 1999).

Variações no ambiente, sejam elas diárias ou sazonais, afetam as funções biológicas de diversas maneiras. Nesse sentido, os dados obtidos através dos estudos bioenergéticos auxiliam a compreender o papel dos organismos nos ecossistemas, pois refletem as interações entre os processos fisiológicos e de alocação de energia com as variáveis ambientais. Esses dados expressam a proporção de energia consumida que é alocada aos vários processos vitais e mostram como esta pode flutuar durante o ano ou ciclo de vida. Desse modo, a bioenergética permite, por exemplo, quantificar as relações entre as taxas de gastos, perdas e produção com as de consumo, permitindo a compreensão das transformações de energia por unidades ecológicas (Phillipson, 1975;
Allen \& Wooton, 1982). Por esses motivos, é amplamente utilizada para investigar as funções de organismos e populações em termos de fluxo de energia entre diferentes níveis tróficos dos ecossistemas marinhos. Dados desse gênero são essenciais para a formulação de modelos ecológicos (Brafield \& Llewellyn, 1982). Além disso, o estudo do orçamento bioenergético de peixes possui ampla aplicabilidade no gerenciamento dos estoques pesqueiros, proteção ambiental, aqüicultura, etc. (Rice, 1990; Adams \& Breck, 1990; Karas \& Thoresson, 1992; Brandt \& Hartman, 1993; Lesser et al., 1997; MacIsaac et al., 1997).

Os gastos de energia com o metabolismo constituem até cerca de $40 \%$ do orçamento, indicando a demanda energética básica do organismo. Para estimar essa demanda, as taxas de consumo de oxigênio podem ser convertidas em energia utilizando-se um equivalente oxicalórico (Qox) (Elliott \& Davidson, 1975; Cech, 1990). Por sua vez, a excreção de compostos nitrogenados constitui um percentual das perdas energéticas do organismo e, também, representa parte da contribuição da ictiofauna na reciclagem de nutrientes em ambientes aquáticos (Du Preez et al., 1990). Ambos os parâmetros são regulados tanto por fatores endógenos, tais como sexo e peso, quanto exógenos, principalmente a temperatura.

Este trabalho tem como objetivo estudar as variações dos gastos energéticos de $H$. steindachneri com os processos metabólicos, através da quantificação das taxas de consumo de oxigênio e excreção de amônia, em função do peso, em diferentes temperaturas, relacionadas às condições oceanográficas locais. Dessa forma, pretende-se contribuir para a compreensão das variações da alocação de energia em relação a fatores preponderantes que regulam o funcionamento do ecossistema. Os dados foram também utilizados para estimar os outros componentes da equação bioenergética, usando o modelo proposto por Du Preez et al. (1990) para teleósteos marinhos, a fim de se obter uma primeira aproximação do orçamento energético de indivíduos jovens, de diferentes tamanhos, dessa espécie.

\section{Material e métodos}

Os peixes foram capturados com o auxílio da embarcação de pesquisa "Veliger II", do IOUSP, em regiões rasas de até $9 \mathrm{~m}$ de profundidade, próximas às praias que circundam a Enseada do Flamengo e a

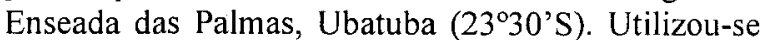
uma rede de arrasto de fundo, com tempo de arrasto de aproximadamente 5 minutos, a fim de evitar-se ao máximo danos físicos causados aos animais. Após a captura, ainda na embarcação, os indivíduos em boas 
condições foram selecionados, imediatamente transferidos para tanques com renovação contínua de água do mar, para serem transportados até a Base Norte "Clarimundo de Jesus" do IOUSP, localizada na Enseada do Flamengo. Na base de pesquisa, os peixes foram colocados em tanques de 500 litros, à salinidade de $35 \%$, com aeração constante, higienização e renovação diária da água do mar, permanecendo em repouso de 5 a 7 dias para aclimatação ao cativeiro. Como suprimento alimentar, forneceram-se camarões frescos, capturados na região, e mantidos vivos em tanques de 500 litros. Após esse período, a alimentação foi suspensa e os peixes foram transferidos para tanques no interior do laboratório com temperatura controlada, onde permaneceram por 48 horas até o início dos experimentos, para aclimatação à temperatura experimental.

Toda água do mar utilizada nos tanques de aclimatação, viveiro de camarões e nos experimentos de laboratório, foi previamente filtrada em filtro tipo CUNO de $1 \mu \mathrm{m}$. Este procedimento minimiza a interferência do metabolismo de microorganismos presentes na água do mar sobre a quantificação das taxas de consumo de oxigênio e excreção de amônia dos peixes.

Para o estudo das variações do metabolismo de rotina (Rr) e da excreção de amônia (Uend) de $H$. steindachneri, foram realizados 259 experimentos em sistema respirométrico de câmaras seladas, em função do peso e do sexo, às temperaturas de $15^{\circ} \mathrm{C}, 20^{\circ} \mathrm{C}$ e $26^{\circ} \mathrm{C}$ e salinidade de $35 \%$. Estes valores estão dentro do intervalo de variação de temperatura da coluna d'água comumente encontrado na zona costeira da região. O metabolismo de rotina e excreção de amônia endógena referem-se àqueles de um animal em jejum, com movimentos calmos e espontâneos. A amostra foi constituída por indivíduos jovens variando de $5,92 \mathrm{~g}$ a $87,84 \mathrm{~g}$ de peso e de $78 \mathrm{~mm}$ a $184 \mathrm{~mm}$ de comprimento.

Os peixes foram colocados individualmente em câmaras respirométricas tubulares de acrílico e mantidos com circulação de água por um período de 3 horas, sendo então o fornecimento de água suspenso e as câmaras seladas. $O$ período de confinamento variou entre 45 e 90 minutos, dependendo do tamanho do animal e do volume do respirômetro, de forma que o nível de oxigênio no interior do respirômetro ao final do experimento fosse sempre mantido acima de $60 \%$ da concentração inicial, a fim de evitar os efeitos da depleção do gás sobre as taxas metabólicas. Amostras de água foram retiradas no início e no final do período de confinamento, para determinação do oxigênio dissolvido pelo método de Winkler (Grasshoff et al., 1983) e para a determinação das concentrações de amônia, pelo método de Koroleff (1970). As diferenças entre as concentrações iniciais e finais do oxigênio e da amônia, multiplicadas pelo volume do respirômetro, descontado o volume do animal (em litros), representam, respectivamente, o consumo e a excreção do animal no período. Os experimentos foram efetuados sempre em um mesmo horário, para minimizar a influência de variações decorrentes dos ciclos diários de luz. Após a mensuração do consumo de oxigênio e da excreção de amônia, os peixes foram sacrificados para as tomadas de medidas de peso úmido, comprimento total e identificação do sexo por inspeção visual das gônadas, segundo Vazzoler (1996). Por esse método, os peixes utilizados foram identificados como jovens, imaturos ou em estágios iniciais de maturação.

Calcularam-se o consumo de oxigênio por indivíduo ( $\mu \mathrm{OO}_{2} /$ hora) e a excreção de amônia por indivíduo ( $\mu$ atgN/hora), em função da temperatura e peso úmido. As relações entre o consumo de oxigênio (Rr) e excreção de amônia (U) com o peso foram descritas pelo logaritmo da equação de regressão Log $(\operatorname{Rr}$ ou $\mathrm{U})=\log \mathrm{a}+\mathrm{b} \log (\mathrm{W})$ (Schmidt-Nielsen, 1999), sendo a e b as constantes que representam a elevação e a inclinação da reta, respectivamente.

Não foram detectadas diferenças significativas nos valores de consumo de oxigênio e de excreção de amônia entre machos e fêmeas. Por esse motivo, as análises foram efetuadas sobre o conjunto total de dados.

Aplicaram-se análises de regressão, para cada caso, utilizando-se todos os dados logaritmizados, sendo calculados os coeficientes de correlação r. Foi aplicada análise de covariância entre as regressões lineares e o teste de comparações múltiplas Newman-Keuls para identificar as diferenças entre as inclinações e elevações dos pares de retas (Zar, 1984).

A partir do consumo de oxigênio de rotina e excreção de amônia, calculou-se o coeficiente de temperatura $Q_{10}$ (Musatov, 1994) para os intervalos de temperatura $15-20^{\circ} \mathrm{C}$ e $20-26^{\circ} \mathrm{C}$. A relação oxigênio:nitrogênio $(\mathrm{O}: \mathrm{N})$ foi determinada pela razão entre as taxas de consumo de oxigênio e excreção de amônia, em cada temperatura (Mayzaud \& Conover, 1988). Os valores do coeficiente de temperatura $Q_{10} e$ da relação $\mathrm{O}: \mathrm{N}$ foram calculados a cada 10 gramas de peso úmido, até 90 gramas.

Para converter os dados de consumo de oxigênio e os de excreção de amônia em calorias (cal/dia), utilizaram-se, respectivamente, o coeficiente oxicalórico de 4,64 cal/ $\mathrm{mlO}_{2}$ (Brett, 1985) e o coeficiente de $5,94 \mathrm{cal} / \mathrm{mgN}$ (Elliot \& Davison, 1975). Para estimar os demais parâmetros bioenergéticos para animais de diferentes tamanhos, a cada temperatura, foi empregada a partição de energia do modelo proposto por Du Preez et al. (1990) para teleósteos marinhos, que considera $\mathrm{C}(100 \%)=\mathrm{F}$ $(10 \% \pm 6)+U(4 \% \pm 1)+\operatorname{Rr}(23 \% \pm 13)+\operatorname{Rd}(21 \% \pm 3)$ $+\mathrm{P}(42 \% \pm 11)$, indicando qual porcentagem da energia consumida pelo peixe $(C)$ é perdida na forma de fezes $(F)$, excreção de produtos nitrogenados $(U)$, 
calor metabólico de rotina $(\mathrm{Rr})$, calor metabólico devido aos processos de digestão (Rd) ou retido como crescimento $(\mathrm{P})$. Foram selecionados para esse procedimento pesos arbitrários de $20 \mathrm{~g} \mathrm{e} 70 \mathrm{~g}$, por corresponderem, aproximadamente, aos indivíduos com menor e maior pesos utilizados nos experimentos e que foram comuns às três temperaturas testadas. Os valores experimentais de $R r$ e $U$ desses animais foram utilizados para inferir os outros componentes da equação.

\section{Resultados}

As médias dos valores de consumo de oxigênio e de excreção de amônia, para cada classe de peso de Haemulon steindachneri, para as três temperaturas, estão graficamente apresentadas nas Figuras 1 e 2.

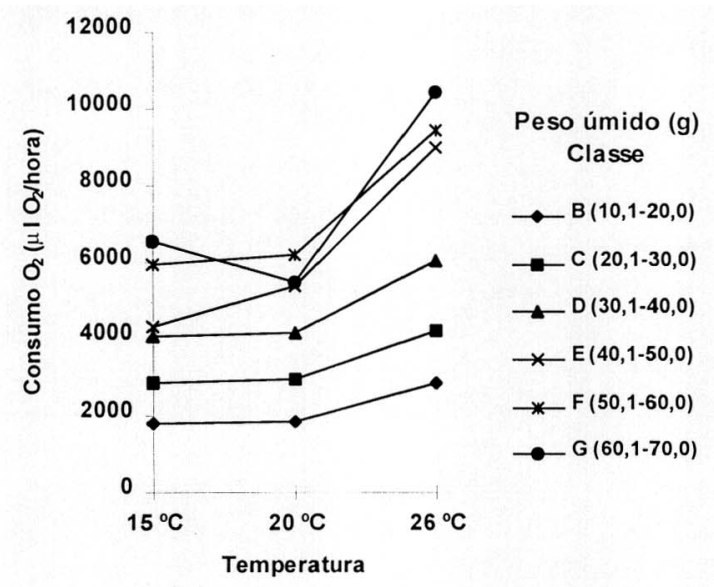

Fig. 1. Médias de consumo de oxigênio de Haemulon steindachneri, de diferentes classes de peso úmido, em função da temperatura.

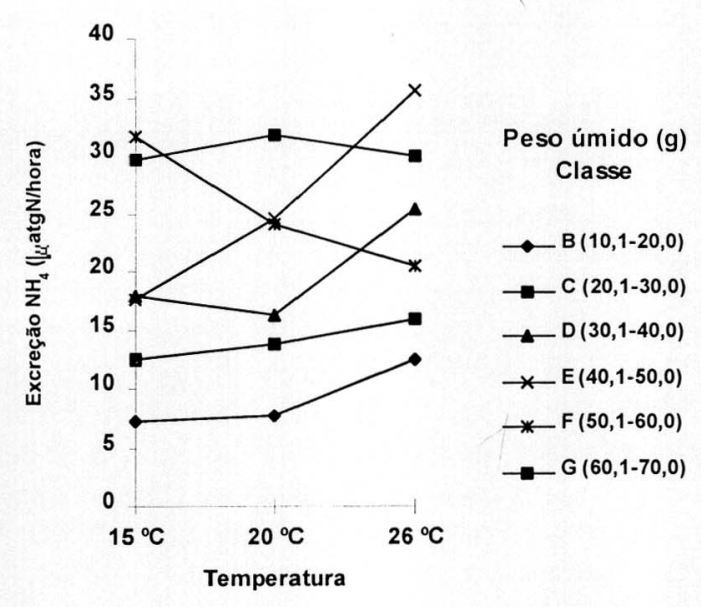

Fig. 2. Médias da excreção de amônia de Haemulon steindachneri, de diferentes classes de peso úmido, em função da temperatura.
O consumo de oxigênio (R) e a excreção de amônia (U) aumentaram com $o$ peso úmido dos peixes (W), em todos os casos. A análise de covariância não detectou diferenças significativas $(p>0,05)$ entre as inclinações (b) das regressões lineares do consumo de oxigênio em função do peso, em nenhuma das temperaturas estudadas. Essa análise, no entanto, indicou diferenças significativas entre as elevações (a). $O$ teste de comparações múltiplas de Newman-Keuls identificou não haver diferenças significativas $(p>0,05)$ entre os valores de a das regressões referentes ao consumo de oxigênio a $15^{\circ} \mathrm{C}$ e $20^{\circ} \mathrm{C}$, demonstrando que nessas temperaturas, este foi semelhante (Fig. 3). Por outro lado, o consumo mais elevado a $26^{\circ} \mathrm{C}$ resultou em diferenças significativas $(p<0,05)$ entre as elevações das regressões a $15^{\circ} \mathrm{C}$ e $26^{\circ} \mathrm{C}$, e a $20^{\circ} \mathrm{C}$ e $26^{\circ} \mathrm{C}$. A excreção de amônia aumentou com a temperatura (Fig. 4). As análises de covariância não indicaram diferenças significativas entre as inclinações $\mathbf{b}$. As comparações das elevações a das regressões lineares demonstraram haver diferenças significativas $(p<0,05)$ entre as três temperaturas testadas.

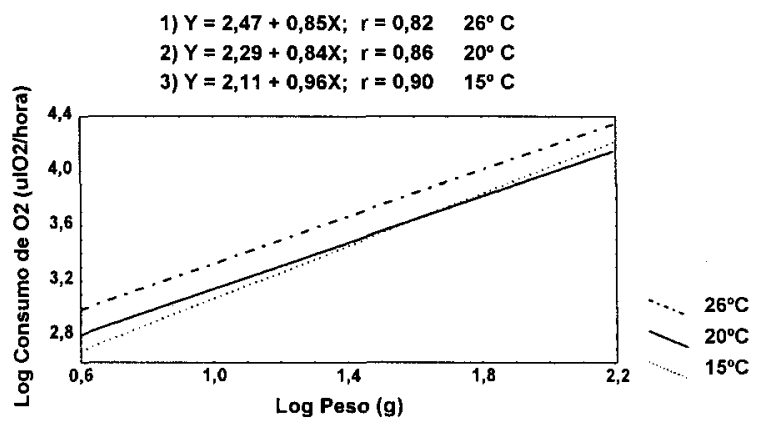

Fig. 3. Regressão linear entre o consumo de oxigênio por indivíduo e o peso úmido de Haemulon steindachneri, nas três temperaturas.

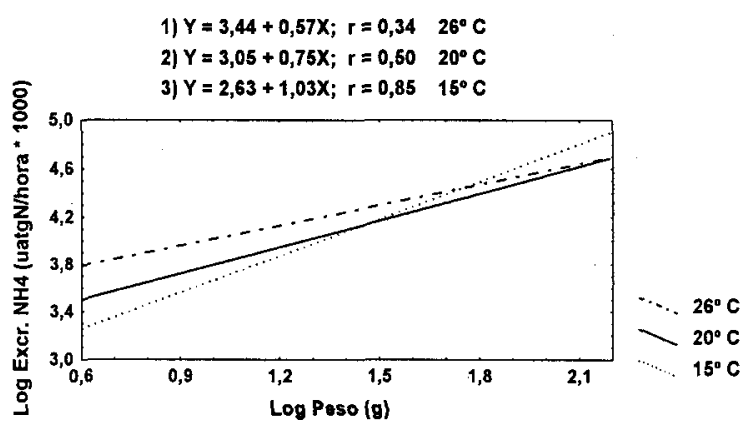

Fig. 4. Regressão linear entre a excreção de amônia por indivíduo $\mathrm{e} o$ peso úmido de Haemulon steindachneri, nas três temperaturas.

Os valores de $Q_{10}$ para o consumo de oxigênio e excreção de amônia estão apresentados na Tabela $1.0 Q_{10}$ do consumo de oxigênio decresceu com o aumento do peso no intervalo $15-20^{\circ} \mathrm{C}$ de 
temperatura, e aumentou no intervalo $20-26^{\circ} \mathrm{C}$. Em relação à excreção de amônia, o $\mathrm{Q}_{10}$ diminuiu em relação ao peso nos dois intervalos de temperatura testados.

Tabela 1. Valores referentes ao coeficiente $Q_{10}$ de temperatura calculados a partir do consumo de oxigênio e excreção de amônia de Haemulon steindachneri, de diferentes pesos.

\begin{tabular}{ccccc}
\hline $\begin{array}{c}\text { Peso } \\
\text { Úmido }(\mathrm{g})\end{array}$ & \multicolumn{3}{c}{$\mathrm{Q}_{10}$} \\
& \multicolumn{2}{c}{ Cons. $\mathrm{O}_{2}$} & \multicolumn{2}{c}{${\text { Excr. } \mathrm{NH}_{4}}$} \\
& $15-20^{\circ} \mathrm{C}$ & $20-26^{\circ} \mathrm{C}$ & $15-20^{\circ} \mathrm{C}$ & $20-26^{\circ} \mathrm{C}$ \\
\hline 10 & 1,32 & 2,07 & 1,91 & 2,24 \\
20 & 1,12 & 2,10 & 1,29 & 1,82 \\
30 & 1,01 & 2,11 & 1,03 & 1,61 \\
40 & 0,95 & 2,12 & 0,88 & 1,48 \\
50 & 0,90 & 2,13 & 0,77 & 1,38 \\
60 & 0,86 & 2,14 & 0,70 & 1,31 \\
70 & 0,83 & 2,14 & 0,64 & 1,25 \\
80 & 0,80 & 2,15 & 0,59 & 1,20 \\
90 & 0,78 & 2,15 & 0,56 & 1,16 \\
\hline
\end{tabular}

Os valores da razão $\mathrm{O}: \mathrm{N}$ (oxigênio:nitrogênio), que indicam o tipo de substrato mais utilizado nos processos catabólicos, estão representados na Tabela 2, para animais entre $10 \mathrm{e}$ $90 \mathrm{~g}$ de peso. Esses valores variaram, respectivamente, entre 17,16 e 14,46 a $15^{\circ} \mathrm{C} ; 15,87$ e 19,60 a $20^{\circ} \mathrm{C}$; e 12,37 e 31,82 a $26^{\circ} \mathrm{C}$.

Tabela 2. Razão oxigênio:nitrogênio (O:N) de Haemulon steindachneri em diferentes temperaturas e pesos.

\begin{tabular}{cccc}
\hline $\begin{array}{c}\text { Peso } \\
\text { (gramas) }\end{array}$ & $15^{\circ} \mathrm{C}$ & $\begin{array}{c}\text { Razão O:N } \\
20^{\circ} \mathrm{C}\end{array}$ & $26^{\circ} \mathrm{C}$ \\
\hline 10 & 17,16 & 15,87 & 12,37 \\
20 & 16,26 & 16,97 & 16,67 \\
30 & 15,75 & 17,64 & 19,84 \\
40 & 15,40 & 18,13 & 22,46 \\
50 & 15,14 & 18,53 & 24,72 \\
60 & 14,92 & 18,85 & 26,73 \\
70 & 14,75 & 19,14 & 28,56 \\
80 & 14,59 & 19,38 & 30,25 \\
90 & 14,46 & 19,60 & 31,82 \\
\hline
\end{tabular}

As estimativas da demanda e das perdas energéticas diárias, por classe de peso, de $H$. steindachneri referentes ao consumo de oxigênio e excreção de amônia em função da temperatura estão apresentadas nas Tabelas 3 e 4 . A demanda energética diária mínima e máxima das classes $\mathrm{B}(10,01-20,00 \mathrm{~g})$ e $\mathrm{G}(60,01-70,00 \mathrm{~g})$ comuns às três temperaturas, foram $199,83 \mathrm{cal} / \mathrm{dia}$ a $15^{\circ} \mathrm{C}$ e $1160,72 \mathrm{cal} / \mathrm{dia}$ a $26^{\circ} \mathrm{C}$, respectivamente. $\mathrm{Da}$ mesma forma, as perdas energéticas diárias variaram de um mínimo de 18,69 $\mathrm{cal} / \mathrm{dia}$ a $15^{\circ} \mathrm{C}$, a um máximo de $76,98 \mathrm{cal} /$ dia a $26^{\circ} \mathrm{C}$.

Tabela 3. Estimativa da demanda energética (cal/dia) em função da temperatura, por classe de peso, a partir das médias do consumo de oxigênio de Haemulon steindachneri.

\begin{tabular}{ccrrr}
\hline Classe & Peso & \multicolumn{3}{c}{ cal/dia } \\
& (gramas) & $15^{\circ} \mathrm{C}$ & $20^{\circ} \mathrm{C}$ & \multicolumn{1}{c}{$26^{\circ} \mathrm{C}$} \\
\hline A & $(0,1-10)$ & $\ldots \ldots \ldots$. & $\ldots \ldots \ldots$ & 129,18 \\
B & $(10,1-20)$ & 199,83 & 205,44 & 319,77 \\
C & $(20,1-30)$ & 316,09 & 331,65 & 467,51 \\
D & $(30,1-40)$ & 454,73 & 461,74 & 672,17 \\
E & $(40,1-50)$ & 479,86 & 597,38 & 1001,05 \\
F & $(50,1-60)$ & 661,26 & 686,32 & 1050,55 \\
G & $(60,1-70)$ & 728,79 & 610,43 & 1160,72 \\
H & $(70,1-80)$ & 1068,57 & $\ldots \ldots \ldots$. & 1094,17 \\
I. & $(80,1-90)$ & 1191,88 & 855,39 & $\ldots \ldots \ldots .$. \\
\hline
\end{tabular}

Tabela 4. Estimativa das perdas energéticas (cal/dia) em função da temperatura, por classe de peso, a partir das médias da excreção de amônia de Haemulon steindachneri.

\begin{tabular}{ccrrr}
\hline Classe & Peso & \multicolumn{3}{c}{$\mathrm{cal} / \mathrm{dia}$} \\
& (gramas) & $15^{\circ} \mathrm{C}$ & \multicolumn{1}{c}{$20^{\circ} \mathrm{C}$} & $26^{\circ} \mathrm{C}$ \\
\hline $\mathrm{A}$ & $(0,1-10)$ & $\ldots \ldots \ldots$ & $\ldots \ldots$. & 23,39 \\
$\mathrm{~B}$ & $(10,1-20)$ & 18,69 & 19,69 & 31,77 \\
$\mathrm{C}$ & $(20,1-30)$ & 31,82 & 35,48 & 40,93 \\
$\mathrm{D}$ & $(30,1-40)$ & 46,01 & 41,80 & 65,10 \\
$\mathrm{E}$ & $(40,1-50)$ & 45,20 & 63,20 & 91,20 \\
$\mathrm{~F}$ & $(50,1-60)$ & 81,13 & 61,77 & 52,76 \\
$\mathrm{G}$ & $(60,1-70)$ & 76,07 & 81,62 & 76,98 \\
$\mathrm{H}$ & $(70,1-80)$ & 98,41 & $\ldots \ldots \ldots$ & $\ldots \ldots$. \\
$\mathrm{I}$ & $(80,1-90)$ & 115,36 & 78,29 & $\ldots \ldots$. \\
\hline
\end{tabular}

A partir desses dados, inferiram-se os demais componentes da equação bioenergética, de acordo com Du Preez et al. (1990) conforme descrito na Tabela 5. Esse procedimento foi adotado para fornecer uma primeira aproximação do balanço energético da espécie, considerando-se indivíduos de diferentes pesos. Os pesos de $20 \mathrm{~g}$ e $70 \mathrm{~g}$ foram selecionados por serem aproximadamente o menor e o maior comuns aos indivíduos utilizados nas três temperaturas estudadas. A extrapolação dos dados de gasto metabólico obtidos em laboratório para valores de necessidade energética de consumo alimentar diário resultou, para peixes de $20 \mathrm{~g}$ e $70 \mathrm{~g}$ respectivamente, em $868,83 \mathrm{cal} / \mathrm{dia}$ e $3168,65 \mathrm{cal} / \mathrm{dia}$, a $15^{\circ} \mathrm{C} ; 893,22 \mathrm{cal} / \mathrm{dia}$ e $2654,04 \mathrm{cal} / \mathrm{dia}$, a $20^{\circ} \mathrm{C}$; e, $1390,30 \mathrm{cal} / \mathrm{dia}$ e $5046,61 \mathrm{cal} / \mathrm{dia}$, a $26^{\circ} \mathrm{C}$. 
Tabela 5. Estimativa do orçamento energético diário, em calorias, de Haemulon steindachneri, para animais de pesos distintos, utilizando-se a alocação de energia do modelo proposto por Du Preez et al. (1990).

\begin{tabular}{|c|c|c|c|c|c|c|c|c|c|c|c|c|}
\hline \multirow{2}{*}{$\frac{\text { Temp. }{ }^{\circ} \mathrm{C}}{15}$} & \multicolumn{2}{|c|}{ Peso (g) C $(100 \%)=$} & \multicolumn{2}{|c|}{$F(10 \% \pm 6)$} & \multicolumn{2}{|c|}{$\mathrm{U}(4 \% \pm 1)$} & \multicolumn{2}{|c|}{$\operatorname{Rr}(23 \% \pm 13)$} & \multicolumn{2}{|c|}{$\mathrm{Rd}(21 \% \mp 3)$} & \multicolumn{2}{|c|}{$P(42 \% \pm 11)$} \\
\hline & 20 & 868,83 & 86,88 & $\pm 52,13$ & 34,75 & $\pm \overline{8,69}$ & 199,83 & $\pm 112,95$ & 182,45 & $\pm 26,06$ & 364,91 & $\pm 95,57$ \\
\hline & 70 & 3168,65 & 316,87 & $\pm 190,12$ & 126,75 & $\pm 31,69$ & 728,79 & $\pm 411,92$ & 665,42 & $\pm 95,06$ & 1330,83 & $\pm 348,55$ \\
\hline 20 & 20 & 893,22 & 89,32 & $\pm 53,59$ & 35,73 & $\pm \overline{8,93}$ & 205,44 & $\pm 116,12$ & 187,58 & $\pm 26,80$ & 375,15 & $\pm 98,25$ \\
\hline & 70 & 2654,04 & 265,40 & $\pm 159,24$ & 106,16 & $\pm 26,54$ & 610,43 & $\pm 345,03$ & 557,35 & $\pm 79,62$ & 1114,70 & $\pm 291,94$ \\
\hline 26 & 20 & 1390,30 & 139,03 & $\pm 83,42$ & 55,61 & $\pm 13,90$ & 319,77 & $\pm 180,74$ & 291,96 & $\pm 41,71$ & 583,93 & $\pm \overline{152,93}$ \\
\hline
\end{tabular}

\section{Discussão}

A quantificação do consumo de oxigênio é um dos meios mais eficazes para estimar os gastos de energia com o metabolismo aeróbico (Becker et al., 1992). Neste trabalho, foi avaliado o metabolismo de rotina, isto é, o consumo de oxigênio do peixe com movimentos livres e espontâneos (Beamish \& Mookherjii, 1964 apud Waller, 1992). Segundo Du Preez et al. (1990), dependendo dos hábitos da espécie, o metabolismo de rotina abrange a maior parte da demanda energética diária de peixes em condições naturais. Os exemplos fornecidos por esses autores podem ser aplicados a Haemulon steindachneri. Estes peixes alimentam-se principalmente de crustáceos e outros organismos bentônicos, sem a necessidade de movimentos intensos freqüentes.

As variações dos gastos metabólicos representam o estado de atividade geral do organismo, que podem ser interpretadas como respostas às variações ambientais e extrapoladas ao nível ecossistêmico (Maclsaac et al., 1997; Parma de Croux, 1997). A determinação das taxas de excreção de amônia por sua vez, contribui substancialmente para as estimativas das perdas energéticas de teleósteos marinhos, além de auxiliar no entendimento do papel dos mesmos na reciclagem de nutrientes no ambiente (Bray et al., 1986; Cockcroft \& Du Preez, 1990; Phan et al., 1993).

Vários sistemas respirométricos podem ser utilizados nas medidas das taxas metabólicas, permitindo a obtenção de dados sob condições experimentais. Esses sistemas diferem em diversos aspectos tais como fluxo de água, tamanho dos tanques, sistemas de controle de qualidade da água, etc. (Rus et al., 2000). Os sistemas respirométricos de câmaras seladas, porém, são ainda muito utilizados em virtude da simplicidade operacional, podendo produzir resultados confiáveis, comparáveis aos demais sistemas, desde que mantidos os cuidados metodológicos essenciais. Entre estes, é necessário evitar o decaimento excessivo da quantidade de oxigênio no respirômetro para que não haja influência da hipóxia e do acúmulo de metabólitos sobre o metabolismo. Por esses motivos, neste trabalho, a concentração de oxigênio dissolvido na água contida no interior da câmara respirométrica no final do período de confinamento foi sempre de no mínimo $60 \%$ em relação à concentração inicial.

Os valores médios de consumo de oxigênio e excreção de amônia do metabolismo de rotina de $H$. steindachneri são compatíveis com os esperados e observados para outros teleósteos marinhos tropicais (Phan et al.,1993; Gomes et al., 1999). O metabolismo pode ser descrito pela equação $R=a W^{b}$, onde $\mathrm{R}$ representa a variação do metabolismo (consumo de oxigênio ou excreção de amônia), W representa a variação do peso úmido e b é o expoente que relaciona as variações metabólicas com o peso. Alguns autores propõem que, em teleósteos, os valores de b variam entre 0,67 e 0,90 para as regressões de consumo de oxigênio e entre $0,47 \mathrm{e}$ 0,99 para as regressões de excreção de amônia, em função do peso úmido (Du Preez et al., 1986; Cockcroft \& Du Preez, 1989; 1990). Apesar dos valores de $\mathbf{b}$ encontrados neste trabalho estarem dentro ou próximos dos limites dos intervalos indicados na literatura, os valores máximos dos expoentes encontrados para as regressões para consumo de oxigênio $(0,96)$ e excreção de amônia $(1,03)$ a $15^{\circ} \mathrm{C}$ são relativamente altos e podem estar refletindo o efeito da temperatura sobre animais de diferentes tamanhos ou, simplesmente, as taxas elevadas de metabolismo da população amostral, constituída principalmente por peixes jovens. De um modo geral, o ecossistema costeiro de Ubatuba é caracteristicamente um local de crescimento de peixes jovens de várias espécies (Rocha \& RossiWongtschowski, 1998). Muitos autores consideram que $o$ expoente $b$ pode variar tanto interespecificamente quanto intra-especificamente, e que valores absolutos não devem ser rigidamente estabelecidos (Hoss, 1967; Gomes et al., 1999).

O sexo do indivíduo pode influenciar na alocação de energia para o metabolismo dos peixes de diversas maneiras, decorrentes das fases do ciclo de vida dos organismos. Em geral, as fêmeas alocam mais energia para o desenvolvimento gonadal, a qual será disponibilizada para as fases iniciais do 
desenvolvimento larval, como substâncias de reserva (Huber \& Bengtson, 1999). Entretanto, neste estudo, não foram detectadas diferenças significativas no consumo de oxigênio e excreção de amônia entre indivíduos machos e fêmeas de $H$. steindachneri. Através da inspeção visual das gônadas verificou-se que estas eram imaturas ou se encontravam em fases iniciais de desenvolvimento, indicando a predominância de indivíduos jovens. $\mathrm{O}$ estudo de peixes de diferentes estágios de maturidade é necessário para verificar se esse resultado se mantém.

A temperatura é um dos principais fatores ambientais que regula 0 conjunto de reações metabólicas em animais ectotérmicos (Burel et al., 1996). Seus efeitos sobre a taxa metabólica dos peixes podem ser observados através da elevação a, desde que as retas sejam paralelas, isto é, que os valores de b não sejam significativamente distintos. Geralmente, a elevação aumenta linearmente com a temperatura, deslocando a reta de regressão em relação ao eixo das ordenadas. Neste trabalho, os valores de $\mathbf{b}$ foram estatisticamente semelhantes entre si, tanto para as regressões de consumo de oxigênio quanto de excreção de amônia, permitindo as análises estatísticas da elevação como forma de estimar as variações metabólicas em função da temperatura e do peso.

As diferenças encontradas no consumo de oxigênio de $H$. steindachneri não foram significativas entre $15^{\circ} \mathrm{C}$ e $20^{\circ} \mathrm{C}$ (Fig. 3), conforme demonstram os valores das elevações a e os de $\mathrm{Q}_{10}$ (Tab. 1). Ocorre, inclusive, um decréscimo no metabolismo a $20^{\circ} \mathrm{C}$, em indivíduos maiores, indicado pelos valores de $\mathrm{Q}_{10}$. Pode-se supor que, nas condições experimentais utilizadas, os animais maiores responderam diferentemente às variações de temperatura do que indivíduos menores. Por outro lado, os efeitos da variação de temperatura são significativos no intervalo de $20-26^{\circ} \mathrm{C}$, com a duplicação da taxa metabólica apontada pelos valores médios de $Q_{10}$, 0 que está dentro do esperado para organismos ectotérmicos. Examinando-se as Figuras 1 e 3 pode-se visualizar claramente essas tendências.

A capacidade de aclimatação a diferentes regimes térmicos varia amplamente entre as espécies. A compensação parcial ou completa do metabolismo aeróbico de animais submetidos à exposição prolongada a uma nova temperatura, após uma mudança abrupta, é comum e muito importante em espécies que experimentam um amplo intervalo de variação no ambiente (Barrionuevo \& Fernandes, 1998). Os dados obtidos neste trabalho sugerem que é provável que ocorra uma faixa de independência térmica entre $15^{\circ} \mathrm{C}$ e $20^{\circ} \mathrm{C}$ nas necessidades energéticas para o metabolismo de $H$. steindachneri. A existência de intervalos de temperatura que aparentemente não afetam a demanda de energia para o metabolismo é um fato relativamente frequiente do ponto de vista biológico (Clarke, 1983; Schmidt-
Nielsen, 1999). Na região de Ubatuba, devido à penetração da Água Central do Atlântico Sul (ACAS) no verão, mais salina e fria do que a Água Costeira (Matsuura, 1986; Castro et al., 1987), os animais da plataforma interna estão expostos a diferenças de temperatura de mais de $10^{\circ} \mathrm{C}$ (de $14^{\circ} \mathrm{C}$ a $24-25^{\circ} \mathrm{C}$ ), entre o fundo e a superfície da coluna d'água. Os indivíduos da espécie estudada são comedores de fundo, capturados na região, desde profundidades superiores a $20 \mathrm{~m}$ até águas rasas. Dessa forma, estão certamente sujeitos a gradientes verticais acentuados de temperatura que podem favorecer estratégias adaptativas no sentido de otimizar a utilização da energia, pelo menos em uma certa faixa de variação. Por esse motivo, portanto, para se verificar com acuidade o efeito da temperatura em peixes, parece importante que se utilizem intervalos experimentais relativamente estreitos, sobretudo quando se estudam indivíduos de espécies sujeitas a ambientes mais instáveis. Caso semelhante foi encontrado para outras espécies de peixes que vivem nessa mesma área (Gomes et al.,1999) e outros grupos de animais de outras áreas, incluindo crustáceos (Phan et al., 1997; 1998).

As taxas de excreção de amônia, por sua vez, são bastante variáveis (Fig. 2) por se tratar de um processo muito complexo sujeito à influência de fatores de difícil controle, tais como tipo de substrato utilizado para processos oxidativos ou estado nutricional (Echevarría et al., 1993; Gomes et al., 1995). Entretanto, apesar dos desvios em relação às médias terem sido relativamente grandes, constatouse que as taxas de excreção de $H$. steindachneri aumentaram significativamente com a temperatura (Fig. 4). A relação O:N (oxigênio:nitrogênio) fornece uma medida que informa o tipo de substrato utilizado nos processos oxidativos para obtenção de energia. Valores entre 3 e 16 indicam a utilização de substrato predominantemente protéico; entre 16 e 60 , indicam um substrato formado por proteínas e lipídeos, e, acima de 60 , indicam o predomínio de lipídeos como substrato para os processos catabólicos (Mayzaud \& Conover, 1988). De um modo geral, os valores da relação O:N indicaram que $H$. steindachneri empregou, como substrato, uma mistura composta por diferentes proporções de proteínas e lipídeos, dependendo da temperatura (Tab. 2). Aos $26^{\circ} \mathrm{C}$, o aumento da relação $\mathrm{O}: \mathrm{N}$, em animais de maior peso, indica a utilização de uma proporção maior de lipídeos, possivelmente devido a maior demanda energética causada pela aceleração do metabolismo (Phan et al., 1997).

Além da temperatura, outros fatores não controlados, tais como fotoperíodo (Karas, 1990; Waller, 1992; Imsland et al., 1995; Huber \& Bengtson, 1999; Boeuf \& Le Bail, 1999), efeito-SDA (Guinea \& Fernandez, 1991) e ritmos biológicos (Liu et al., 1997; Thetmeyer, 1997; Kim et al., 1998) podem afetar a variabilidade dos processos vitais. Por 
esse motivo, os experimentos com $H$. steindachneri para estudo dos efeitos da temperatura foram realizados sempre em um mesmo horário ou período, tendo sido os animais mantidos em jejum por 48 horas. Em geral, observa-se que $H$. steindachneri é um peixe de hábitos noturnos, abrigando-se durante o dia nas áreas rochosas e coralinas, deixando essas estruturas ao entardecer rumo a regiões de fundos inconsolidados, a procura de alimento (Estrada, 1986). Por esse motivo, estudos sobre a influência do ciclo de luz na alocação de energia da espécie são fortemente recomendados.

Os valores mensurados de gasto metabóliço de rotina diário conforme apresentado na Tabela 3, indicam que $H$. steindachneri tem uma demanda relativamente elevada quando comparada às de outras espécies de teleósteos da região (Phan et al., 1993; Gomes et al., 1999), possivelmente por se tratar de uma espécie com estrutura corpórea robusta, aparentemente com massa muscular desenvolvida. Ao se compararem os dados da Tabela $3 \mathrm{com}$ os da Tabela 4, constata-se que as perdas energéticas com - catabolismo protéico endógeno representa aproximadamente $10 \%$ da demanda energética do metabolismo de rotina. Todos os valores, entretanto, estão dentro dos esperados para teleósteos em geral (Brett \& Groves, 1979).

As inferências dos parâmetros bioenergéticos realizadas neste trabalho (Tab. 5) tiveram como intuito oferecer uma primeira aproximação das necessidades energéticas desses animais, baseando-se nas medidas de laboratório de apenas dois de seus componentes. As variações dos dados refletem, portanto, apenas as que ocorreram com as medições efetivamente realizadas. Dessa forma, a acuidade dos valores não pode absolutamente ser avaliada, a menos que se realizem experimentos para esse fim. No entanto, a partição de energia proposta pelos autores do modelo foi estabelecida a partir de medidas obtidas com várias espécies de peixes carnívoros costeiros, principalmente jovens, características essas que correspondem aos indivíduos utilizados neste trabalho. Pode-se, portanto, supor que os valores obtidos fornecem pelo menos uma inferência da ordem de magnitude dos fenômenos. Dados de campo e de laboratório, juntamente com os obtidos neste trabalho, contribuirão para o estabelecimento da alocação de energia específica para a espécie estudada e de suas variações em função de fatores ambientais.

\section{Agradecimentos}

Agradecemos $o$ apoio do Instituto Oceanográfico da USP pelas facilidades oferecidas e ao Conselho Nacional para o Desenvolvimento Científico e Tecnológico ( $\mathrm{CNPq})$ pelas bolsas concedidas (Procs. 133380/98-0, 301480/91-0 e 300299/77-0).

\section{Referências bibliográficas}

Adams, S. M. \& Breck, J. E. 1990. Bioenergetics. In: Schreck, C. B. \& Moyle, P. B. eds. Methods of fish biology. Bethesda, American Fisheries Society. p.389-415.

Aidar, E.; Gaeta, S. A.; Gianesella-Galvão, S. M. F.; Kutner, M. B. B. \& Teixeira, C. 1993. Ecossistema costeiro subtropical: nutrientes dissolvidos, fitoplâncton e clorofila- $a$ e suas relações com as condições oceanográficas na região de Ubatuba, SP. Publção esp. Inst. oceanogr., S Paulo, (10):9-43.

Allen, J. R. M. \& Wooton, R. J. 1982. The effect of ration and temperature on the growth of threespined stickleback, Gasterosteus aculeatus L. J. Fish Biol., 20(4):409-422.

Barrionuevo, W. R. \& Fernandes, M. N. 1998. Time-course of respiratory metabolic adjustments of a South American fish, Prochilodus scrofa, exposed to low and high temperatures. J. appl. Ichthyol., 14(1-2):37-41.

Becker, K.; Meyer-Burgdorff, K. \& Focken, U. 1992. Temperature induced metabolic cost in carp, Cyprinus carpio L., during warm and cold acclimatization. J. appl. Ichthyol., 8(1-4):10-20.

Boeuf, G. \& Le Bail, P. Y. 1999. Does light have an influence on fish growth? Aquaculture, 177(1-4):129-152.

Brafield, A. E. \& Llewellyn, M. J. 1982. Animal energetics. London, Blackie Publishers. 306p.

Brandt, S. B. \& Hartman, K. J. 1993. Innovative approaches with bioenergetics models: future applications to fish ecology and management. Trans. Am. Fish. Soc., 122(5):731-735.

Bray, R. N.; Purcell, L. J. \& Miller, A. C. 1986. Ammonium excretion in a temperate-reef community by a planktivorous fish, Chromis punctipinnis (Pomacentridae), and potencial uptake by young giant kelp Macrocystis pyrifera (Lamminariales). Mar. Biol., 90(3):327-334.

Brett, J. R. 1985. Correction in use of oxicalorific equivalent. Can. J. Fish. aquat. Sci., 42(7):13261327. 
Brett, J. R. \& Groves, T. D. D. 1979. Physiological energetics. In: Hoar, W. S.; Randall, D. J. \& Brett, J. R. eds. Fish Physiology. New York, Academic Press, 8:279-352.

Burel, C.; Person Le-Ruyet, J.; Gaumet, F.; Le Roux, A.; Séverè, A. \& Boeuf, G. 1996. Effects of temperature on growth and metabolism in juvenile turbot. J. Fish Biol., 49(4):678-692.

Burke, N. C. 1995. Nocturnal foraging habitats of French and bluestriped grunts, Haemulon flavolineatum and $H$. sciurus, at Tobacco Caye, Belize. Environ. Biol. Fishes, 42(4):365-374.

Castro Filho, B. M.; Miranda, L. B. \& Miyao, S. Y. 1987. Condições hidrográficas na Plataforma Continental ao largo de Ubatuba: variações sazonais e em média escala. Bolm Inst. oceanogr., S Paulo, 35(2):135-151.

Cech Jr., J. J. 1990. Respirometry. In: Schreck, C. B. \& Moyle, P. B. eds. Methods for fish biology. Bethesda, American Fisheries Society. p.335-362.

Clarke, A. 1983. Life in cold water: the physiological ecology of polar marine ectotherms. Oceanogr. mar. Biol. a. Rev., 21:341-453.

Clarke, R. D. 1999. Diets and metabolic rates of four Caribbean tube blennies, genus Acanthemblemaria (Teleostei: Chenopsidae). Bull. mar. Sci., 65(1):185-199.

Cockcroft, A. C. \& Du Preez, H. H. 1989. Nitrogen and energy loss via nonfaecal and faecal excretion in the marine teleost Lithognathus lithognathus. Mar. Biol., 101(3):419-425.

Cockcroft, A. C. \& Du Preez, H. H. 1990. Nitrogen and energy loss in the marine teleost Lithognathus mormyrus (Linnaeus). J. expl mar. Biol. Ecol., 140(3):159-171.

Cunningham, P. T. M. 1983. Estudo comparativo da ictiofauna da costa oeste e Enseada das Palmas da Ilha Anchieta, Enseada do Flamengo e Enseada da Fortaleza (Lat. 23 $23^{\circ} \mathrm{S}-23^{\circ} 33^{\prime} \mathrm{S}$, Long. $45^{\circ} 03^{\prime} \mathrm{W}-45^{\circ} 09^{\prime} \mathrm{W}$ ) Ubatuba, Estado de São Paulo - Brasil. Tese de doutorado. Universidade de São Paulo, Instituto Oceanográfico. 133p.

Du Preez, H. H.; McLachlan, A. \& Marais, J. F. K. 1986. Oxygen consumption of a shallow water teleost, the spotted grunter, Pomadasys commersonni (Lacépéde, 1802). Comp. Biochem. Physiol., 84A (1):61-70.
Du Preez, H. H.; McLachlan, A.; Marais, J. F. K. \& Cockcroft, A. C. 1990. Bioenergetics of fishes in a high-energy surf-zone. Mar. Biol., 106(1):1-12.

Echevarría, G.; Zarauz, N.; López-Ruiz, J. \& Zamora, S. 1993. Study of nitrogen excretion in the gilthead seabream (Sparus aurata L.): influence of nutritional state. Comp. Biochem. Physiol., 105A (1):17-19.

Elliott, J. M. \& Davison, W. 1975. Energy equivalents of oxygen consumption in animal energetics. Oecologia, 19(3):195-201.

Estrada, M. R. 1986. Habitos alimentarios de los peces del genero Haemulon (Pisces: Haemulidae) de los arrecifes de la region de Santa Marta, Colombia. An. Inst. Investnes mar., Punta Betin, (15/16):49-66.

Furia, R. R. 1996. Composição das capturas de peixes na Enseada das Palmas - Ilha Anchieta, Ubatuba SP: variabilidade espaço - temporal. Dissertação de mestrado. Universidade de São Paulo, Instituto Oceanográfico. $147 \mathrm{p}$.

Gomes, V.; Phan, V. N. \& Passos, M. J. A. C. R. 1995. Estudo do metabolismo de rotina e excreção de amônia do anfípoda antártico Waldeckia obesa em duas temperaturas distintas. Bolm Inst. oceanogr., S Paulo, 43(2):129-139.

Gomes, V.; Phan, V. N.; Passos, M. J. A. C. R. \& Forneris, L. L. C. 1999. Oxygen consumption and ammonia excretion of the searobin Prionotus punctatus (Scorpaeniformes, Triglidae) at two different temperatures. Rev. bras. oceanogr., 47(2):127-136.

Grasshoff, K.; Ehrhardt, M. \& Kremling, K. 1983. Methods of seawater analysis. $2^{\text {nd }}$ ed. Weinheim, Verlag Chemie. 419p.

Guinea, J. \& Fernández, F. 1991. The effect of SDA, temperature and daily rhythm on the energy metabolism of the mullet Mugil saliens. Aquaculture, 97(4):353-364.

Hoss, D. E. 1967. Rates of respiration of estuarine fish. In: ANNUAL CONFERENCE OF THE SOUTHERN ASSOCIATION OF GAME AND FISH COMMISSIONERS, 21. New Orleans, 1967. Proceedings. New Orleans, SAGFC. p.416423.

Huber, M. \& Bengtson, D. A. 1999. Effects of photoperiod and temperature on the regulation of the onset of maturation in the estuarine fish Menidia berylina (Cope) (Atherinidae). J. expl. mar. Biol. Ecol., 240(2): 285-302. 
Imsland, A. K., Folkvord, A. \& Stefansson, S. O. 1995. Growth, oxygen consumption and activity of juvenile turbot (Scophthalmus maximus L.) reared under different temperatures and photoperiods. Neth. J. Sea Res., 34(1-3):149-159.

Karas, P. 1990. Seasonal changes in growth and standard metabolic rate of juvenile perch, Perca fluviatilis L. J. Fish Biol., 37(6):913-920.

Karas, P. \& Thoresson, G. 1992. An application of a bienergetics model to Eurasian perch (Perca fluviatilis L.). J. Fish Biol., 41(2):217-230.

Kim, W. S.; Kim, J. M.; Kim, M. S.; Park, C. W. \& Huh, H. T. 1998. Effects of sudden changes in salinity on endogenous rhythms of the spotted sea bass Lateolabrax sp. Mar. Biol., 131(2):219-225.

Koroleff, F. 1970. Direct determination of ammonia in natural waters as indophenol blue. ICES Interlab. Rep., 3:19-22.

Lesser, M. P.; Martini, F. H. \& Heiser, J. B. 1997. Ecology of hagfish, Myxine glutinosa L. in the Gulf of Maine. I. Metabolic rates and energetics. J. expl mar. Biol. Ecol., 208(1-2):215-225.

Liu, H.; Sakurai, Y. \& Munehara, H. 1997. Diel rhythms of oxygen consumption and activity level of juvenile flounder Paralichthys olivaceus. Fish. Sci., 63(5): 655-658.

Lokkeborg, S. \& Fernös, A. 1999. Diel activity pattern and food search behaviour in cod, Gadus morhua. Environ. Biol. Fishes, 54(3):345-353.

MacIsaac, P. F.; Golf, G. P. \& Speare, D. J. 1997. Comparison of routine oxygen consumption rates of three species of pleuronectids at three temperatures. J. appl. Ichthyol., 13(4):171-176.

Matsuura, Y. 1986. Contribuição ao estudo da estrutura oceanográfica da região sudeste entre Cabo Frio (RJ) e Cabo de Santa Marta Grande (SC). Ciência e Cult., S Paulo, 38(8):1439-1450.

Mayzaud, P. \& Conover, R. J. 1988. O:N atomic ratio as a tool to describe zooplankton metabolism. Mar. Ecol.-Progr. Ser., 45(3):289-302.

McFarland, W. N.; Ogden, J. C. \& Lythgoe, J. N. 1979. The influence of light on the twilight migrations of grunts. Environ. Biol. Fishes, 4(1):9-22.

Menezes, N. A. \& Figueiredo, J. L. 1980. Manual de peixes marinhos do sudeste do Brasil. IV. Teleostei (3). Universidade de São Paulo, Museu de Zoologia. 47p.
Meyer, J. L. \& Schultz, E. T. 1985. Migrating haemulid fishes as a source of nutrients and organic matter on coral reefs. Limnol. Oceanogr., $30(1): 146-156$.

Musatov, A. P. 1994. The van't-Hoff temperature coefficient of energy metabolism in lower vertebrates. Hydrobiol. J., 30(5):96-99.

Ogden, J. C. \& Ehrlich, P. R. 1977. The behaviour of heterotypic resting schools of juvenile grunts (Pomadasydae). Mar. Biol., 42(3):273-280.

Parma de Croux, M. J. 1997. Relationship between metabolic rate and body weight in Salminus maxillosus (Valenciennes, 1840). J. Aquacult. Trop., 12(1):17-22.

Phan V. N.; Gomes, V.; Carvalho, P.S.M. \& Passos, M. J. A. C. R. 1997. Effect of body size, temperature and starvation on oxygen consumption of Antarctic krill Euphausia superba. Rev. bras. oceanogr., 45(1/2):1-10.

Phan V. N.; Gomes, V.; Morais, D. V. \& Passos, M. J. A. C. R. 1993. Estudo bioenergético de animais marinhos costeiros. I. Paralonchurus brasiliensis (Perciformes, Sciaenidae). Publção esp. Inst. oceanogr., S Paulo, (10):199-215.

Phan V. N.; Gomes, V. \& Passos, M. J. A. C. R. 1998. Routine metabolism and ammonia excretion of the Antarctic amphipod Bovallia gigantea (Pfeffer, 1988) (Crustacea, Amphipoda) in two distinct temperatures. Pesq. antart. bras., 3(1):7785.

Phillipson, J. 1975. Introduction to ecological energetics. IBP Handbook, 24:3-14.

Quinn, T. P. \& Ogden, J. C. 1984. Field evidence of compass orientation in migrating juvenile grunts (Haemulidae). J. expl mar. Biol. Ecol., 81(2):181192.

Rice, J. A. 1990. Bioenergetics modeling approaches to evaluation of stress in fishes. Am. Fish. Soc. Symp., 8:80-92.

Rocha, M. L. C. F. 1997. Variação na composição e na abundância da ictiofauna ao longo de 24 horas, na Enseada das Palmas, Ilha Anchieta (SP). Dissertação de mestrado. Universidade de São Paulo, Instituto Oceanográfico. 108p.

Rocha, G. R. A. \& Rossi-Wongtschowski, C. L. D. B. 1998. Demersal fish community on the inner shelf of Ubatuba, southeastern Brazil. Rev. bras. oceanogr., 46(2):93-109. 
Rus, A. S.; Enjuto, C.; Morales, A. E.; Hidalgo, M. C. \& García-Galleno, M. 2000. Description of a facility for studying energy metabolism in fish: application to aquaculture. Aquacult. Engin., 21(3):169-180.

Saul; A. C. 1999. Dinâmica da comunidade de peixes em recifes artificiais nos entornos do Parque Estadual da Ilha Anchieta, Ubatuba, SP. Tese de doutorado. Universidade de São Paulo, Instituto Oceanográfico. $178 \mathrm{p}$.

Schmidt-Nielsen, K. 1999. Fisiologia animal: adaptação e meio ambiente. $5^{\text {a }}$ ed. São Paulo, Santos Editora. 600p.

Thetmeyer, H. 1997. Diel rhythms of swimming activity and oxygen consumption in Gobiusculus flavescens (Fabricius) and Pomatoschistus minutus (Pallas) (Teleostei: Gobiidae). J. expl mar. Biol. Ecol., 218(2):187-198.
Vazzoler, A. E. A. M. 1996. Biologia da reprodução de peixes teleósteos: teoria e prática. Maringá, EDUEM. 169p.

Waller, U. 1992. Factors influencing routine oxygen consumption in turbot, Scophthalmus maximus. J. appl. Ichthyol. 8(1-4):62-71.

Zar, J. H. 1984. Biostatistical analysis. $2^{\text {nd }}$ ed. Englewood Cliffs, N. J., Prentice-Hall. 718p.

(Manuscrito recebido 07 novembro 2000; revisado 28 maio 2001; aceito 31 outubro 2001) 